\title{
HISTORIA DEL DEPARTAMENTO DE QUÍMICA DE LA UNIVERSIDAD PEDAGÓGICA NACIONAL
}

\author{
Rafael Humberto Ramírez Gil \\ Profesor Universidad Pedagógica Nacional
}

\begin{abstract}
The Universidad Pedagógica Nacional (National Pedagogycal University) was created andacademically and administratively organized, according to Law 3153, 1968. The Chemistry Department emerged as a basic academic unit con firmed by the Board of Directors during it's regular session held on July $24^{\text {th }}, 1969$, as established in the meeting record number 12.
\end{abstract}

The man Department Objectives, by that time, were:

- Prepare highly academic qualified teachers in the Chemistry field to teach high school and university level.

- Promote research work in teaching methods as well as in basic science.

- Pro vide advisory services to the National Education Ministry and other institutions requiring them.

The above objectives aimed at the development of educational research and community extension functions which every university must accomplish.

\section{RESUMEN}

Con base en el Decreto 3153 de 1968, se organizó la Universidad Pedagógica Nacional tanto académica como administrativamente, apareciendo el Departamento de Química como una de las unidades académicas básicas, hecho que fue refrendado por el Consejo Directivo de la época en sesión ordinaria del 24 de julio de 1969, Acta No.12 de esa fecha. Los objetivos iniciales del Departamento fueron inicialmente los siguientes:

- Formar profesores de alto nivel académico en el área de la química para la enseñanza a nivel medio y superior.

- Fomentar la investigación tanto en docencia como en ciencia básica.

- Prestar asesoría al Ministerio de Educación Nacional y a entidades e institutos que la solicitaran.

Estos objetivos permitieron cumplir con las funciones de formación, investigación y extensión de toda universidad.

Para tener una idea de lo que ha sido el Departamento de Química de nuestra Universidad, es necesario recordar el origen de nuestra alma mater cuya historia se remonta al año de 1928, cuando en la Universidad de Tunja y por iniciativa del pedagogo RAFAEL BERNAL JIMENEZ, se inicio la preparación de docentes con un nivel académico superior al que se estaban preparando los futuros docentes en las Escuelas Normales que formaban institutores. 
Con la llegada de los pedagogos alemanes JULIUS SIBER y FRANCISCA RADKE, se produjo un avance en la formación de educadores, preparando un grupo muy importante de profesores colombianos con conocimientos más avanzados en las áreas de Ciencias Naturales y matemáticas, como en Educación.

Posteriormente en 1931 se creó anexa a la Universidad Nacional de Colombia la llamada Facultad de Educación, constituyéndose en la primera unidad académica de carácter docente, con independencia, a nivel superior, mediante decreto 1990 de 1933.

En 1936, por la Ley 30, la Facultad se separa de la Universidad nacional y recibió el nombre de Escuela Normal Superior, dependiendo directamente del Ministerio de Educación, habiendo sido su primer rector el Doctor JOSÉ FRANCISCO SOCARRAS, quien la impulsé creando una gran mística entre el alumnado que a poco tiempo se convirtieron en los pioneros de la educación en el país y la institución en la líder de los procesos educativos que produjeron grandes cambios sociales.

En 1951, por Decreto Ley 192, se le cambió el nombre por el de Escuela Normal Universitaria, la cual fue fraccionada mediante Ley 1955 de! mismo año en la sección femenina de Bogotá y la sección masculina con sede en Tunja. En ambas sedes funcionaron programas de licenciatura conformados por disciplinas científicas y campos educativos que tenían que ver con la formación integral de un profesor. Estos programas fueron agrupados en facultades entre la que figuraron: La Facultad de Matemáticas y Física, Biología y Química, Filología e Idiomas, Ciencias Sociales y Económicas y Facultad de Educación, estructura que se conservé hasta cuando cada seccional tuvo vida jurídica independiente.

Al producirse la división mencionada, el Ministerio de Educación Nacional, produjo la Resolución $\mathrm{N}^{0} 3383$ del 2 de diciembre de 1952, solicitada por la Doctora Francisca Radke. Esta Resolución se refiere a la reglamentación para la adjudicación de becas en la "Escuela Normal Femenina" que funciono en el Instituto Pedagógico Nacional del cual había sido nombrada rectora la Doctora Radke. Durante los 2 años siguientes hubo una serie de Decretos que le cambiaban el nombre a la institución, maremagnun que se resolvió en 1955 cuando el gobierno nacional produjo el Decreto del 10 de febrero creando la Universidad Pedagógica Nacional Femenina con sede en Bogotá, nombre que fue cambiado por el de Universidad Pedagógica Nacional Bogotá, mediante el Decreto 2188 del 2 de agoste de 1962.

El Decreto 3153 del 26 de diciembre de 1968 bajo la presidencia del doctor Carlos Lleras Restrepo, organizó la Universidad consignándose en su articulado la definición, naturaleza, objetivos, funciones, administración y patrimonio de la institución. En desarrollo de éste Decreto y bajo la rectoría del Doctor Jaime Sanín Echeverry en 1969, se realizó un seminario profesoral que propuso una estructura de la Universidad basada en una Facultad de Educación y los Departamentos de Matemáticas, Física, Biología, Química, Humanidades, Español, Lingüística y Educación Física, naciendo así el Departamento de Química. Con base en estos estudios, los Consejos Directivos y Académicos de la época, redactaron un Reglamento Académico que fue aprobado por el Consejo Directivo en la sesión del 24 de julio de 1969, según Acta No. 12 de esa fecha, reglamento que fue aprobado con carácter experimental para el II Semestre de 1969, razón por la cual, su aprobación no se hizo por Acuerdo.

El parágrafo 3 del Artículo 9 del precitado Reglamento define lo que es un Departamento: "Entenderse por Departamento la Unidad Académica que agrupa el 
personal universitario que a través de la docencia, la investigación y la extensión, se dedica al estudio de una ciencia, o un arte, o de asignaturas afines, desde los niveles elementales hasta los más avanzados, de manera integral, teniendo en cuenta las necesidades curriculares de la institución”. De igual manera el Artículo 11, establece las funciones de cada Departamento.

\section{DEPARTAMENTO DE QUIMICA}

El Departamento de Química, se consolidó e inicio sus labores propias a partir del 1 de enero de 1970, con el nombramiento del primer jefe y la integración de la planta de personal docente y administrativo integrada por 1 jefe, 4 profesores de tiempo completo, 2 catedráticos, 1 jefe de laboratorio y una secretaria.

Se inició la estructura del Departamento, analizando la problemática educativa del momento, pasando a la estructuración del plan de estudios conducentes a la formación de un buen Licenciado en Química, que además de ser un buen profesor para el nivel de enseñanza media debería tener otra perspectiva, como la proseguir estudios a nivel maestría o doctorado, dándole además, algunos instrumentos que le permitieran al menos tener alguna idea de la aplicación de la química a la industria. Fueron 3 los objetivos principales de éste plan de estudios:

1. Formar profesores de alto nivel académico en el área de Química para a enseñanza a nivel medio y superior.

2. Iniciar e incrementar la investigación tanto en docencia como en ciencias básicas.

3. Prestar asesoría al Ministerio de Educación Nacional y a entidades e institutos que lo requieran y capacitar docentes en ejercicio (programas de extensión)

Los tres objetivos permitían cumplir con las principales funciones de toda institución universitaria, la formación, la investigación y la extensión hoy llamada promoción social, campos que no ha dejado de atender el Departamento en sus 29 años de existencia.

Como producto de la evaluación institucional de 1969, se vio la necesidad de introducir cambios muy notorios en el sistema tradicional de formación de profesionales de la educación, influenciados por el pensamiento norteamericano tanto en lo estructural como en lo académico. Fue la Filosofía INEM, la que marcó el horizonte en el diseño del currículo del Departamento de Química en su primera etapa, caracterizado por su flexibilidad curricular basada en objetivos que permitían formar a un profesor, que además de instructor, fuera un investigador. Existía el servicio de Consejería Académica para orientar a los estudiantes en la selección de su propio plan de estudios y en sus métodos de trabajo. Así mismo cada profesor de planta, además de atender a sus alumnos en asuntos relacionados con su cátedra, dirigía los Centros Sociales con grupos de 15 y 20 estudiantes con quienes realizaba actividades culturales, los fines de semana.

La programación académica se inició con el régimen semestral y la unidad de trabajo era el crédito que equivalía a 48 horas de trabajo semanal de un estudiante dentro de las cuales se contaban las horas aula, las horas para tareas o ejercicios, la preparación de conferencias, la investigación bibliográfica y el estudio independiente. 
El programa académico de pregrado conducía al título de Licenciado en Educación con estudios principales en Química o Mayores en Química y mayores en otra área como matemáticas o Mayores en Química y menores en otra área. Para obtener el título de Licenciado, el estudiante debía completar por lo menos 128 créditos que un alumno de tiempo completo podía cursarlos en 8 semestres como mínimo.

El Departamento de Química fue la primera unidad académica de la Universidad Pedagógica Nacional que inició el proceso de investigación tanto en investigación básica como en investigación en pedagogía y didáctica incluyendo en su primer plan de estudios el seminario llamado "Introducción a la Investigación" que fue desarrollado inicialmente por 4 profesores y posteriormente consolidado con la aparición de las monografías de pregrado dirigidas por todos los profesores de planta. Actualmente hay cientos de monografías, no todas de la misma altura académica pero son el reflejo de que nuestros egresados llevan un ingrediente investigativo que los distingue de los egresados de otras universidades con los mismos programas. El énfasis de la investigación está en el área de Pedagogía y Didáctica.

Este primer programa de Licenciatura en Educación con estudios básicos, mayores o principales en una o dos de las áreas, fueron aprobadas por Resolución N" 2022 del 24 de abril de 1975 del MEN.

En 1980, con el Decreto Ley 80 del mismo año, se reglamenté la Educación Superior, norma que estableció que los programas universitarios conducentes a la formación de profesores, darían el título de Licenciado en la disciplina correspondiente, aspecto que fue ratificado en el Artículo 3 del Decreto Reglamentario 2725 de 1980. Por esta razón, el programa que actualmente ofrece el Departamento a nivel de Pregrado es el Licenciatura en Química, codificado en el ICFES con el No. 43909.

El Decreto Ley 80 llevó a una reforma Académica de los programas y a una reforma en la estructura administrativa, creándose 3 grandes facultades; la de Ciencia y tecnología, Artes, Humanidades y Educación. La Facultad de Ciencia y Tecnología formada por los Departamentos de: Biología, Física, Matemáticas, Educación Física, Química y Tecnología; administra programas de formación universitaria y de postgrado en campos afines del conocimiento.

El programa de Licenciatura en Química está regido por los principios académicos generales, establecidos en el Acuerdo 072 de junio 22 de 1982 emanado del Consejo Directivo de la Universidad orientado "sus actividades académicas a la formación de profesionales de la Educación con capacidad creadora, crítica e investigativa, sólidos conocimientos en la disciplina particular"; respetando los principios de libertad de cátedra y de aprendizaje. La investigación adquirió mayor impulso orientándose a generar conocimientos y a facilitar los procesos de enseñanza y aprendizaje, a suscitar en el estudiante un espíritu innovador, creativo y critico. En el Departamento se han impulsado la investigación en Pedagogía y didáctica de la Química y un poco en la investigación básica.

El nuevo plan de estudios del programa de Licenciatura está conformado por 4 áreas académicas: El Area de Formación Pedagógica y Didáctica, el área de formación específica, el área de integración y el área flexible conformada por actividades artísticas, estéticas, culturales y deportivas. 
Para medir la actividad académica, se opté por las unidades de labor Académica ULAS, en sus 3 tipos A, B, C, que sumadas debía dar un mínimo de 3.200 para optar el Título de Licenciado. En cuanto a programas de EXTENSIÓN, el Departamento de Química ha organizado diferentes cursos de capacitación de profesores en ejercicio del nivel medio Universitario. Ha organizado seminarios a nivel nacional e internacional para tratar temas como la investigación, la enseñanza de las ciencias y otros. Así mismo sus profesores hemos participado en eventos nacionales e internacionales de carácter científico y pedagógico. Se han desarrollado pasantías internacionales especialmente con el programa de maestría. Se ha prestado asesoría universidades, Secretarias de Educación y varios colegios.

A la industria, se presta el servicio de análisis Químico con muy buenos resultados.

\section{NUEVA REFORMA}

A partir de la autonomía universitaria consagrada en la Constitución de 1991 y las directrices establecidas en la Ley General de Educación, la ley 30 de 1992 sobre Educación Superior, el Decreto 272 de 1998 así como las normas internas de la Institución y los criterios de docentes y dicentes del Departamento, se está pensando en una nueva reforma del programa de Licenciatura en Química, en donde habrá una estructura curricular por ciclos, una evaluación por competencias y una concepción filosófica teniendo como principio fundamental la investigación.

En publicaciones, cabe destacar la labor de varios profesores que han publicado libros sobre enseñanza de las Ciencias y en Ciencias Naturales así como en Química para el nivel de bachillerato, etc. Se han publicado muchos trabajos producto de investigación, en revistas nacionales e internacionales.

Es importante destacar que varios profesores del Departamento han coadyuvado al desarrollo de la universidad y de la educación del país, ocupando puestos directivos como Rectores Encargados, Vicerrectores Académicos, Decanos, Jefes de Departamento, Coordinadores de Programa, Directores de Institutos, etc.

\section{MAESTRÍA EN DOCENCIA DE LA QUÍMICA}

Con la reforma académica de 1980, se creó el programa de Maestría en Docencia de la Química, aprobado por el Acuerdo del ICFES N $N^{0} 115$ del 26 de mayo de 1983, codificado ante el mismo Instituto con el $\mathrm{N}^{0} 63905$.

Mediante convenio $N^{0} 1106$ con la Universidad Pedagógica y Tecnológica de Colombia de Tunja, se replicó este programa en dicha universidad con autorización del ICFES, mediante Resolución No. 2630 del 15 de octubre de 1992, entidad que le dio el mismo código 63905.

El objeto primordial de éste programa es el desarrollo de la investigación en la pedagogía y el aprendizaje de la química, buscando desarrollar actitudes científicas e investigativas en los participantes, formando pensadores de la Química capaces de generar nuevos conocimientos. 
La estructura curricular contemplaba una fundamentación teórica, una fundamentación técnica, la investigación y las actividades complementarias.

El programa se inició con 2 líneas; la línea 1 de "Investigación de Problemas Pedagógicos y didácticos en el aprendizaje de conceptos en Química' y la línea 2 "Contribuciones a la enseñanza de la química. Mediante la caracterización química del ambientes naturales colombianos".

Cada una de estas líneas estaban compuestas de una serie de seminarios que permitían profundizar en contenidos científicos desarrollando simultáneamente su pedagogía y didáctica. Posteriormente las dos líneas se fusionaron y los seminarios se reestructuraron. En 1996, el consejo Académico reestructuré el programa, quedando el que hoy está funcionando.

La metodología de los seminarios fue inicialmente la del seminario Alemán y posteriormente se complementé con el taller quedando como una nueva metodología el seminario-taller. Hasta el momento se han tenido 11 promociones en Bogotá y 4 en Tunja. Los alumnos están distribuidos en casi todas las universidades del país y en muchos colegios de bachillerato. Es un programa reconocido internacionalmente siendo uno de los primeros que se abrió en América Latina.

\section{DOCTORADO EN EDUCACIÓN. ÁREA EDUCACIÓN EN CIENCIAS NATURALES}

Mediante convenio Interinstitucional 139-93, entre las universidades de Antioquia, Valle, Nacional de Colombia, Industrial de Santander y Pedagógica Nacional, se logró iniciar un programa Interinstitucional de doctorado en Educación con diferentes áreas, siendo una de ellas la de Educación en Ciencias Naturales que fue asignada a la Universidad Pedagógica Nacional, por cuanto el comité Interinstitucional de Doctorado en Educación (CIDE), consideró que en el Departamento de Química existía una infraestructura y una experiencia por el programa en Maestría, que facilitaba el desarrollo del programa, razón por la cual se solicité al profesor Fidel Antonio Cárdenas Salgado diseñara y coordinara el programa.

El Decreto 2791 del 22 de diciembre de 1994 reglamenta los programas de Doctorado. El Acuerdo 031 del 14 de julio de 1995 emanado del Consejo Superior de la Universidad Pedagógica Nacional, creó en la Universidad el programa Interinstitucional de Doctorado en Educación, área Educación en Ciencias. Mediante Acuerdo 003 del 17 de enero de 1996, el Consejo Superior aprobó las actividades académicas del programa. El Consejo Superior en su sesión extraordinaria del 6 de agosto de 1997, Acta 17 delegó a la Rectoría la expedición de las orientaciones académico-administrativas del programa, las cuales fueron adoptadas por Resolución Rectoral N ${ }^{0} 1453$ del 9 de diciembre de 1997.

A nivel nacional el programa fue aprobado por Resolución del MEN y codificado en el ICFES bajo el No.43909.

En nuestra universidad inició labores académicas el 8 de octubre de 1997 con 8 alumnos en sus 5 líneas de investigación. 


\title{
PROFESORES
}

PROFESORES DE PLANTA OUE HAN PRESTADO SUS SERVICIOS AL DEPARTAMENTO DE QUIIMICA DESDE 1970 HASTA LA FECHA

\author{
Alfaro Pérez Agapito \\ Ardua Prieto Julia Elvira \\ Camargo Castro Rafael \\ Caicedo López Humberto \\ Castellanos Tapias Miguel Arturo \\ Cárdenas Salgado Fidel Antonio \\ Erazo Parga Manuel Antonio \\ Gallego Badillo Rómulo \\ Gómez Cruz Rigoberto \\ Guerrero Santafé Jaime Enrique \\ González Páez Rafael Eduardo \\ Ladino Ospina Yolanda \\ Perilla Díaz Aurora del Carmen \\ Pórez Miranda Roymán \\ Pinzón Mora Guillermo \\ Pinzón Rodriguez Mauro \\ Ramírez Gil Rafael Humberto \\ Salcedo Torres Luis Enrique \\ Zapata Castañeda Pedro Nel
}

\section{JEFES DEL DEPARTAMENTO DE QUÍMICA}

Ramirez Gil Rafael Humberto (4 veces)

Pinzón Mora Guillermo

Alfaro Pérez Agapito (E)

Perilla Díaz Aurora del Carmen

Caicedo López Humberto (2 veces)

Pérez Miranda Roymán

Guerrero Santafé Jaime Enrique $(E)$

Salcedo Torres Luis Enrique

Erazo Parga Manuel Antonio

Cárdenas Salgado Fidel Antonio

Gómez Cruz Rigoberto (E)

Gallego Badillo Rómulo

Pinzón Rodríguez Mauro (E)

\section{BIBLIOGRAFIA}

Cardona de Mejía, A. 1973. La Universidad Pedagógica Nacional. Un estudio sobre su evolución a través de las normas legales. Documentación Educativa UPN. Vol. $2 \mathrm{~N}^{0} 3$.

Ramírez, Gil R. H. 1981. Los 10 años del Departamento de Química de la Universidad Pedagógica Nacional. Revista ENLACE año $11 \mathrm{~N}^{0} 3$ Departamento de Química. UPN. 
Universidad Pedagógica Nacional. 1969. Reglamento Académico. Acta $\mathrm{N}^{0} 12$ del Consejo Directivo.

1982. Reglamento Académico Acuerdo $\mathrm{N}^{0} 072$. 